\title{
1918 und der Beginn des wissenschaftlichen Braindrain aus Österreich
}

This article looks at the history of braindrain from Austria and shows that is was not only an effect of the "Anschluss" 1938. It already began with the increase of antisemitism after the collapse of the Habsburg Empire: Nobel prize winners and young academic talents left the country permanently. Many stayed for some time. However: At least they had to leave university, supplying key contributions by non-university research. There is only a small number of academics still known today who were able to make it to university professorships, e.g. Hans Kelsen (1919), Karl Bühler (1922), Moritz Schlick (1922), Nikolai Turbetzkoy (1922), Hermann Mark (1932), Erwin Schrödinger (1936). If they had not passed away or left the country before the "Anschluss", they also became victims of NS-persecution and expulsion. While the countries of refuge treated their competence as a gain, in Austria the academics excluded from the universities and/or expulsed from the country were not recognized as a loss. The path into provincialism taken at the Austrian universities in 1945 and the time after was first adopted in 1918.

"Österreichs verheerendes Migrationssaldo" lautet der Titel eines entlarvenden Berichts in der „Neuen Zürcher Zeitung“ vom 1. April 2014. Der zunehmende Ausverkauf der Wissenschaft wird hier auf eine „Fehlentwicklung im System" - Klientelpolitik und Reformstau zurückgeführt. ${ }^{1}$ Braindrain ohne Braingain hat in Österreich eine lange Tradition, schon um 1900 verlor es seine besten Köpfe. Anlässlich der Wiedereinführung des 1898 abgeschafften Kollegiengeldes 1919 wurden die Gründe hierfür öffentlich debattiert: Universitätsprofessoren verdienten weniger als Verwaltungsbeamte, und auch in der Ausstattung der Hörsäle, Labore und Bibliotheken hielt Österreich keineswegs mit Deutschland Schritt, was zeigte, „wie wenig Wert und Gewicht die österreichischen Regierungen der Wissenschaft, ihrer freien Lehre und ihrer praktischen Anwendung beilegten. “2

\footnotetext{
${ }^{1}$ Kattinger, Migrationssaldo 25.

2 SPERL, Wiedereinführung 3.
}

Mit dem Zerfall der Monarchie 1918 nahm die Abwanderung der Intelligenz zu. Die Gründe hierfür waren hausgemacht, nämlich ein viel zu geringer Staatsaufwand für Wissenschaft und Forschung, der längst vor dem "Anschluss" 1938 zum Ausschluss der jungen Talente aus der Universität führte. ${ }^{3}$ Ihnen stand die Option offen auszuwandern oder neben einem Brotberuf außeruniversitär Wissenschaft zu treiben.

Bemerkenswert ist, dass die dauerhafte Wirtschaftskrise und der Stellenabbau zwar wesentliche Gründe für den Ausschluss vieler junger Talente waren, aber nicht die zentrale Ursache. Im Rückblick lässt sich diese in einer „Fehlentwicklung im System" von Universität und Politik erkennen: Universität und Unterrichtsver-

\footnotetext{
${ }^{3}$ Vgl. POSCH, Ingrisch, Dressel, "Anschluß" und Ausschluss. Zum Hochschulwesen in der Zwischenkriegszeit vgl. WEINZIERL, Hochschulleben 72-85. LICHTENBERGER-FENZ, Hochschulpolitik. HÖFLECHNER, Wissenschaft 57-74. KONRÁD, Geisteswissenschaften 193-218.
} 
waltung hatten die mit dem UOG 1873 definitiv verrechtlichte Autonomie durch amtlich praktizierten Antisemitismus und "Vetternschaftsund Günstlingswirtschaft" missbraucht: ${ }^{4}$ erklärungsbedürftige Lehrstuhlbesetzungen, verhinderte Habilitationen und rassistische Studentenordnungen (,Volksbürgerschaftsprinzip“) sind ein Indiz dafür, dass die universitäre Selbstverwaltung nicht mehr zur Sicherung der Wissenschaftsfreiheit und zukunftsweisender, voraussetzungsloser Forschung eingesetzt wurde. ${ }^{5}$

Wer von den jungen Forschenden Österreich nicht verließ, fand in der Hauptstadt außerhalb der Universität eine vergleichsweise blühende Wissenschaftslandschaft vor: private Akademikerzirkel (wie den Kreis um Hans Kelsen), Wissenschaftsvereine (wie den Verein Ernst Mach) und außeruniversitäre Institute von Weltrang (wie das Institut für Konjunkturforschung, die Biologische Versuchsanstalt, das Institut für Radiumforschung). In Bezug auf Wien lässt sich für diese Zeit von einer dualistischen Struktur der Wissenschaft - Universität und außeruniversitäre Forschung - sprechen. Darauf haben u.a. Friedrich Engel-Janosi, Ludwig Mises oder Friedrich August Hayek aufmerksam gemacht, deren differenzierte Betrachtung folgendes zeigt: Während die Universität Wien seit 1918 junge Talente (m.E.) systematisch aus politischen und "rassischen" Gründen ausgrenzte und damit dem Braindrain durch Abwanderung Vorschub leistete, bewog das außeruniversitäre Tätigkeitsfeld viele als Linke oder Juden angefeindete Forscherinnen und Forscher noch eine Zeitlang zum Verbleib.

In dieser Abhandlung wird der Braindrain aus Österreich nach 1918 vor dem Hintergrund dieser dualistischen Struktur untersucht und im Überblick dargestellt. Zu zeigen sein wird:

\footnotetext{
${ }^{4}$ ANONYM, Koalition 2.

${ }^{5}$ Vgl. Feichtinger, Verletzte Autonomie [im Druck]
}

- Erstens, dass der Talenteschwund an der Universität Wien mit dem Zerfall der Monarchie radikal zunahm. Ursachen waren zunehmender „rassischer" Antisemitismus, verletzte Autonomie und Wissenschaftsnationalismus in einem längst international verschränkten Wissenschaftssystem verbunden mit Wissenschaftsfeindlichkeit, die Stellenabbau nach sich zog;

- Zweitens, dass zwischen der Abwanderung der universitär und der außeruniversitär verankerten Intelligenz Unterschiede $\mathrm{zu}$ bemerken sind. An der Universität korrelierte die Abwanderung mit den Kündigungswellen: 1934 verloren die liberal, sozialdemokratisch oder nationalsozialistisch gesinnten Professoren ihre Stellen, 1938 wurden Juden, Vertreter des autoritären Ständestaats und solche, die als politisch unzuverlässig eingestuft wurden, vertrieben. Was die außeruniversitäre Wissenschaftskultur betrifft, verliefen Abwanderung und Braindrain kontinuierlicher: Viele verließen Österreich weit vor 1938, kaum einer kehrte zurück;

- Drittens, dass - wie Mitchell G. Ash zeigt Wissenschaftsösterreich den „Weg in die Provinzialität [...] nicht erst 1938, sondern viel früher" einschlug;6 dass 1938 - wie Friedrich Stadler zeigt - die universitäre und außeruniversitäre „Wissens- und Wissenschaftskultur" durch die "Vertreibung der Vernunft" nachhaltig zerschlagen wurde,7 und dass 1945 zwei Kontinuitäten, jene zum autoritären Ständestaat und nach einer Latenzzeit - jene zum Nationalsozialismus stärker waren, als der Wille zum Bruch mit der jüngeren Vergangenheit und zur Zurückholung der zwischen 1918 und 1938 verdrängten und vertriebenen Talente. Christian Fleck spricht in diesem Zusammenhang von der „autochthonen Provinzialisierung".8

\footnotetext{
${ }^{6}$ AsH, Jüdische Wissenschaftlerinnen und Wissenschaftler 93-122.

7 STADLER, Vertriebene Vernunft 9-41.

${ }^{8}$ FLECK, Autochthone Provinzialisierung 67-92.
} 
Sie hat in Österreich Tradition: Der Aufstieg junger, als Juden eingestufter Wissenschaftler zur ordentlichen Professur war schon um 1900 erschwert. Die Statistik dazu lieferte 1907 der frisch gewählte Abgeordnete Josef Redlich, der selbst seit 1901 als Jurist habilitiert war, in einer Rede im Reichsrat: An der juridischen Fakultät der Universität Wien vermerkte er unter den 15 Ordinarien vier ,Nichtarier' (zwei davon getauft); an der philosophischen Fakultät unter den 48 ordentlichen Professoren drei ,Nichtarier', an der medizinischen Fakultät unter 23 Ordinarien zwei jüdischer Herkunft. Sei das die vielbesagte "Judenherrschaft"? Er konstatierte, dass die besten Köpfe „nach der an der Universität und auch im Unterrichtsministerium herrschenden Auffassung nicht mehr unterzubringen" seien. Kein Wunder, aber schade, dass Österreich seine Spitzenforscher „ins Ausland gehen“ ließe. So "unpraktischer Antisemitismus" würde an den preußischen Universitäten nicht geübt. ${ }^{9}$ Nach dem Zerfall der Monarchie verschärfte sich die Lage: Juden wurde zunehmend auch die Habilitation und damit jede Aufstiegschance verwehrt.

Thema dieser Abhandlung ist die rassistisch motivierte Verdrängung der Talente aus der Universität, die sich in wirtschaftlicher Not als ,national', "deutsch' und ,katholisch' verstand. Die Folge davon war ein regelrechter wissenschaftlicher Braindrain durch Abwanderung aus Österreich ab 1918.

\section{Universität und Anschlussfrage}

Am 26. Oktober 1918 bekannte sich der Senat der Universität Wien in einer außerordentlichen

\footnotetext{
${ }^{9}$ Rede im Haus der Abgeordneten am 4. 12. 1907, im Rahmen der Debatte über den Dringlichkeitsantrag Masaryks vom 22. 11. 1907 betreffend die Garantien für die Lehr- und Lernfreiheit an den Universitäten, StenProt AH, 40/XVIII, 2938-2945; vgl. REDLICH, Situation 281-283 (= Vorlage zur Rede).
}

Sitzung „rückhaltlos zu dem neuen Staatswesen“. Wenige Monate später, Anfang Februar 1919, sprach er sich allerdings „in einer einstimmigen Kundgebung“ für den "Anschluß Deutschösterreichs an Deutschland" aus. ${ }^{10}$ Die Anschlussoption wurde trotz des „Unglücksakts von St. Germain" von der Wiener Universität während der Ersten Republik offiziell verfolgt. ${ }^{11}$ 1925 beschloss der dritte ordentliche Hochschultag zu Wien auf Anregung der Wiener rechtsund staatswissenschaftlichen Fakultät und der Rektorenkonferenz in Verbindung mit der Professoren- und Studentenschaft einstimmig ein Memorandum, das sich die staatliche Vereinigung von Österreich mit dem Deutschen Reich zum Ziel setzte. ${ }^{12}$

Die Universität Wien arbeitete dem "Anschluss" durch Angleichung der Studiensysteme aktiv vor. So wurden z.B. die akademischen Grade wechselseitig anerkannt; in Wien wurden das staatswissenschaftliche Doktorat nach deutschem Vorbild eingeführt und ein deutschösterreichischer Wissenschaftsfonds gegründet; viele Wiener Hörer traten dem Verband der "Deutschen Studentenschaft" bei, der auf dem ersten deutschen Studententag in Würzburg 1919 auf großdeutscher und völkischer Grundlage gegründet worden war; 1925 wurde in Wien der Deutsche Hochschul- und Studententag mit Anschlusskundgebung (1925) abgehalten, 1931 in Graz.

Schon vor 1918 stand fest, dass die Universität Wien „mit den gleichartigen Anstalten des Deutschen Reiches in engster Beziehung" bleiben musste, um als selbständiger, aber gleichwertiger Teilhaber "die Höchstwerte deutscher Geistesarbeit den nichtdeutschen Völkern" nahezubringen „und tiefere Einsichten in die Kulturgrundlagen dieser wiederum dem deutschen

\footnotetext{
${ }^{10}$ BECKE, Bericht 7.

${ }^{11}$ SCHWIND, Bericht 9.

12 Vgl. SPERL, Bericht 18.
} 
Volke“ zu vermitteln. ${ }^{13}$ Nach 1918 wurde diese Zusammenarbeit unter dem Vorzeichen des erhofften "Anschlusses" gepflegt: „In der wechselseitigen Angleichung unserer und der reichsdeutschen Einrichtungen erblicken wir unser Heil“, verkündete Prorektor Friedrich Johann Becke 1918/19, „hierin hoffen wir uns nach wie vor als Glied des deutschen Gesamtvolkes zu bewähren."14 1921 zeigte sich der Jurist Ernst Schwind in einem Artikel in der „Neuen Freien Presse“ über „die Steigerung der Wechselbeziehungen, die Betätigung des Gedankens der inneren Einheit der deutschen Wissenschaft" erfreut; allerdings war er auch über „die Aufsaugung unserer Besten und Größten durch den benachbarten großen Freund" besorgt, ${ }^{15}$ waren doch die vakanten Lehrstühle u.a. mit Wiener Professoren besetzt worden.

\section{Universität in Not}

Im März 1921 hieß es in der "Arbeiter-Zeitung": „Die Wiener Universität verblutet sich. [...] Die großen Lehrer schwinden einer nach dem anderen dahin. Unsere berühmte Stätte wissenschaftlicher Tätigkeit verödet. [...] Was Wunder, daß jede Berufung nach Deutschland, nach der Schweiz für unsere Hochschullehrer unwiderstehlich wird!"16 Im Studienjahr 1920/21 hatten u.a. die Chemiker Wilhelm Schlenk und Hans Fischer die Wiener Universität verlassen: ${ }^{17}$ „Ein Gelehrter nach dem anderen geht inzwischen ab“, schrieb Hans Kelsen 1921 in der „Neuen Freien Presse“. Die von Kelsen vorgeschlagenen

\footnotetext{
${ }^{13}$ ReISCH, Aufgaben 86f.

${ }^{14}$ BECKE, Bericht 7f.

${ }^{15}$ SCHWIND, Berufungen 1.

${ }^{16}$ ANONYM, Koalition 2.

${ }^{17}$ Im Studienjahr 1923/24 verließ der ordentliche Professor für politische Ökonomie Carl Grünberg Wien. Grünberg wurde zum Gründungsdirektor des Instituts für Sozialforschung in Frankfurt am Main ernannt.
}

Studiengebühren für „zahlungskräftige Ausländer" sollten die Notlage der Wiener Professoren lindern, deren Realeinkommen weniger als die Hälfte des Einkommens der Kollegen in Deutschland und der Tschechoslowakei betrug. ${ }^{18}$ Der Ausweg wirtschaftlicher Selbstverwaltung wurde allerdings durch die Politik verstellt, die Not vorübergehend durch internationale Spenden gelindert. 1921 wurde der „Verein der Freunde der Wiener Universität“, im Studienjahr 1921/22 der „Verein zur Erhaltung der Universität Wien“ gegründet. ${ }^{19}$ Die Vereine sollten neben Vertretern von Industrie und Wirtschaft sowie Mäzenen wie dem Ehepaar Jerome und Margarethe Stonborough, der Schwester Ludwig Wittgensteins, Franz Boas und der "Emergency Society in Aid of European Science and Arts" sowie dem "Rockefeller Foundation International Health Board" beträchtliche Mittel zur Rettung der Universität zur Verfügung stellen. ${ }^{20}$ Aufgrund der Forderung der deutschen Studentenschaft der Universität Wien, Juden den Zugang zu akademischen Ämtern zu verwehren, die Zahl der Lehrenden jüdischer Abstammung auf $10 \%$ zu beschränken und den numerus clausus für jüdische Studenten einzuführen, drohte der „Verein zur Erhaltung der Universität" im Jahr 1922, „solange keine Unterstützungen zukommen zu lassen, als der akademische Senat nicht eine ,befriedigende Stellung' gegenüber der Forderung nach dem Numerus clausus einnehme. [...] Es ginge nicht an, daß von Juden geleistete Spenden Hetzern zugute kommen."21 Im Studienjahr 1920/21 wurde von deutschvölkischer Seite das „Deutsche Hilfswerk für die Hochschulen Deutschösterreichs" nach dem Vorbild der Berliner „Notgemeinschaft der

\footnotetext{
${ }^{18}$ KELSEN, Not 1.

${ }^{19} \mathrm{Vgl}$. ANONYM, Erhaltung 3.

${ }^{20}$ Vgl. BECKE, Bericht; SCHWIND, Bericht; DOPSCH, Bericht; DIENER, Bericht; SPERL, Bericht; WeTTSTEIN, Hilfsaktionen 2.

${ }^{21}$ ANONYM, Drohungen 6.
} 
deutschen Wissenschaft" errichtet. ${ }^{22}$ Seit 1929 wurden der "Österreichisch-deutschen Wissenschaftshilfe" von Berliner Seite beachtliche Mittel zur Verfügung gestellt mit dem Ziel „der Aufrechterhaltung der gemeinsamen Kultur“ ${ }^{\prime 23}$

\section{Die Ausländer- und Judenfrage}

Die Universität Wien war eine der ersten Massenuniversitäten. Im Wintersemester 1918/19 erreichte die Studierendenzahl die Höhe von 10.515 Hörern und übertraf damit sogar die Höchstziffer vor 1914.24 In den ersten drei Kriegsjahren war ein Rückgang von 6.900 Studierenden $\mathrm{zu}$ verzeichnen gewesen, obwohl 1914/15 mehr als 1.500 Studierende der Universitäten Krakau [Kraków], Lemberg [Lwiw, Lwów] und Czernowitz [Tscherniwzi, Cernăuți, Czerniowce] nach Wien ausgewichen waren. Im Studienjahr 1915/16 war somit nahezu jeder zweite Student der Universität Wien galizischer oder bukowinischer Herkunft. Zu Kriegszeiten waren diese Studierenden noch als Gewinn verbucht worden, danach verschärfte sich die Haltung der Universität ihnen gegenüber radikal im Ton: „Ein Abflauen der jüdischen Hochflut an den Wiener Hochschulen ist noch nicht zu bemerken gewesen“, hieß es 1919 in der "Reichspost". Die Besuchszahlen zeigten "das erschreckende Übergewicht der fremdrassigen Elemente über die einheimischen Studierenden erst recht.“" 25 Somit bestehe eine "sehr bedrohliche Gefahr für den deutschen Charakter der Wiener

\footnotetext{
${ }^{22}$ DOPSCH, Bericht 13; DIENER, Bericht 19.

${ }^{23}$ Vgl. FENGLER, LUXBACHER, Österreichisch-Deutsche Wissenschaftshilfe 303-328. Protokoll über die Sitzung des Kuratoriums der Österreichisch-deutschen Wissenschaftshilfe in Wien am 22.12.1929, Archiv der ÖAW, Wissenschaftshilfe, Kart. 1, 2.

${ }^{24} \mathrm{Vgl}$. POSCH, Studierende 61-97.

${ }^{25}$ ANONYM, Rückgang 7.
}

Universität und die Ausländerfrage [...] [sei] vorwiegend [eine] Judenfrage ${ }^{\text {“. }}{ }^{26}$

Friedrich Johann Becke, Wiener Universitätsrektor 1918/19, sah in der "ganz unverhältnismäßigen Stärke“ des „Einschlags von Studenten aus Galizien, Russisch-Polen und der Bukowina" eine Erklärung und zugleich ein Argument für den sich radikalisierenden Judenhass an der Universität Wien: „Das sind Ziffern“, so der prononcierte Antisemit Becke, „die es begreiflich machen, daß der bodenständige Teil der Studentenschaft gegen diese Zuwanderung Abwehr versuchte. Auch das übereinstimmende Urteil vieler Universitätslehrer geht dahin, daß unter diesem Teil der Studentenschaft eine große Zahl schlecht vorbereiteter festgestellt werden muß, was eine Herabdrückung des wissenschaftlichen Niveaus [...] zur notwendigen Folge hat. ${ }^{\prime 27}$

Diese Art von Antisemitismus war nicht neu: 1876 war Theodor Billroth für seine öffentliche Zurückstufung galizischer Medizinstudenten wegen ihres angeblich andersartigen Bildungshintergrundes von den Wiener Studenten als Held verehrt worden. ${ }^{28}$ Anfang der 1920er Jahre wurden die Anstrengungen der Universität, Juden auszuschließen, ähnlich gewürdigt. Zu Jahresende 1922 unterstützte Karl Diener, Rektor der Universität Wien und Antisemit, das erwähnte Memorandum der Studentenschaft zur Einführung eines numerus clausus von $10 \%$ für jüdische Studierende und Lehrende. In der „Reichspost" schrieb er: „In der geradezu erschreckenden Invasion solcher rassen- und wesensfremder Elemente, deren Kultur, Bildung und Moral tief unter jener der bodenständigen deutschen Studentenschaft stehen, liegt der wahre Krebsschaden unserer akademischen Verhältnisse. Der Abbau der Ostjuden muß heu-

\footnotetext{
${ }^{26}$ M. ST., Die obenan sind 7.

27 BECKE, Bericht $11 \mathrm{f}$

${ }^{28} \mathrm{Vgl}$. AsH, Jüdische Wissenschaftlerinnen und Wissenschaftler 102. RATHKOLB, Gewalt und Antisemitismus an der Universität Wien 71-85.
} 
te im Programm jedes Rektors und Senats einer deutschen Hochschule einen hervorragenden Platz einnehmen. Der fortschreitenden Levantisierung Wiens muß wenigstens an den Hochschulen Einhalt geboten werden.“29

Drei Dinge lassen aufhorchen: In Bezug auf das Ziel des Abbaus jüdischer Lehrender beklagte Diener, dass „infolge der geltenden Habilitationsvorschriften ausschließlich die wissenschaftliche Qualifikation für die Habilitation eines Privatdozenten maßgebend sein darf und nach dem Staatsgrundgesetz die Zugehörigkeit zu einer bestimmten Konfession kein Hindernis für die Erreichung eines allen Staatsbürgern zugänglichen Amtes bildet."30 In Bezug auf den numerus clausus stellte Diener in einem weiteren Artikel in der „Deutschösterreichischen Tageszeitung" unmissverständlich fest, dass er seine „Durchführung für möglich und begrüßenswert halte, soweit es sich um jüdische Hörer ausländischer Staatszugehörigkeit" handelte. Schließlich antizipierte er in Bezug darauf, wer Jude sei, schon 1922 die NS-Definition: „Allein in der Forderung der deutschen Studentenschaft ist auch nicht die Zugehörigkeit zur mosaischen Konfession gemeint, sondern eine Beschränkung jener Lehrenden verlangt, die jüdischer $\mathrm{Ab}$ stammung sind. “31 Den Protest der Wiener Kultusgemeinde im Bundeskanzleramt erwiderte die deutsche Studentenschaft in einem offenen Brief, in dem sie die Kultusgemeinde mahnte, sich nicht „zum Schutzpatron aller Juden, welchen Staates auch immer" zu erheben, weil sie damit „den Beweis erbrachte, daß die jüdischen Glaubensgenossen eben ein Volk" seien. In Österreich sei aber nicht "das jüdische Volk", sondern nur "die jüdische Religion" anerkannt. ${ }^{32}$ Damit zeigte sich klar und deutlich, dass der Antisemitismus ein „rassischer" geworden war.

\footnotetext{
${ }^{29}$ DIENER, Memorandum 1.

${ }^{30}$ Ebd.

${ }^{31}$ DIENER, Vorgänge 2.

${ }^{32}$ ANONYM, Offener Brief 5.
}

Im November 1920 hatten Rektor Schwind und der Senat an der Universität Wien kurzerhand den numerus clausus eingeführt, ihn aber wieder zurücknehmen müssen. Den nächsten Vorstoß Rektor Dieners und der deutschen Studentenschaft im Dezember 1922 erwiderte der Senat durch den Hinweis auf seine Unzuständigkeit; er erklärte aber „daß durch die häufige Ansässigmachung landfremder Elemente auch der nationale Charakter der Wiener Universität berührt" würde, und Universität und Politik aufzufordern, „geeignete Maßregeln zu ergreifen, um Ausländern, deren Bildungsgrad dem Niveau der Wiener Universität nicht entspricht, von ihr fernzuhalten.“33 1923 wurde ein neuerlicher Vorstoß der Studenten vom Senat mit dem Argument gesetzlicher Hindernisse zurückgewiesen.

In den Chor antisemitischer Hetze der Studierenden und Professoren stimmten auch maßgebliche Politiker ein: 1920 bezeichnete Dr. Ignaz Seipel die Zuwanderung ostjüdischer Studenten als Hauptursache für die Überfüllung der Hochschulen: "Abwehrmaßregeln" müssten getroffen, der numerus clausus eingeführt werden, um die „ohnehin schon große Gefahr" auszuräumen, „daß gewisse akademische Berufe zu einer ausschließlichen Domäne der Juden werden." Als Bundeskanzler sollte sich Seipel von diesem "Notwehrantisemitismus" distanzieren ${ }^{34}$ und sich den Angriffen durch den Standpunkt entziehen, „daß die staatsbürgerlichen Rechte aller Bundesbürger, welcher Religion und Konfession sie seien, gewahrt werden müßten". ${ }^{35}$

Die Zahl jüdischer Studierender verringerte sich auch ohne offiziellen numerus clausus seit dem Studienjahr 1922/23 erheblich; ihr Anteil halbier-

\footnotetext{
33 ANONYM, Antwort 1.

34 SEIPEL, Kulturpolitik 2.

${ }^{35}$ ANONYM, Bundeskanzler 6.
} 
te sich zwischen 1920/21 und 1925/26.36 Ursache dafür waren die zunehmend radikaleren antisemitischen Ausschreitungen sowie ein versteckter numerus clausus: Zum Studium waren nur noch Absolventen von Staatsgymnasien zugelassen, deren Besuch den Juden im Zarenreich verboten gewesen war. ${ }^{37}$ Zugleich wurden die Aufnahmebedingungen für ausländische Hörer verschärft: Sie zahlten die fünffachen Studiengebühren von Inländern. ${ }^{38}$

\section{Universität und Talenteschwund}

An der Universität Wien häufte sich schon in der Zwischenkriegszeit der Talenteschwund. Viele Jungwissenschaftlerinnen und Jungwissenschaftler, denen der ,Makel' jüdischer Herkunft und/oder politischer und wissenschaftlicher Unverträglichkeit mit der an der Universität vorherrschenden Weltanschauung anhaftete, wurden kontinuierlich aus der Universität verdrängt. Durch verhinderte Habilitationen und die Nichtberufung in Wien tätiger Spitzenforscher (wie z.B. Ludwig von Mises oder Joseph Schumpeter) verlor die Universität Wien im Inund Ausland an Ansehen. 1934 wurde sie vornehmlich politisch, 1938 politisch und „rassisch“ gesäubert.

Schon um 1918 hatten zahlreiche als Juden diffamierte Spitzenforscher die Universität verlassen, u.a. der Chemiker Fritz Paneth, der Sprachforscher Leo Spitzer, die Mediziner Robert Bárány und Karl Landsteiner sowie (rund ein Jahrzehnt später) der Soziologe Friedrich Hertz. Mit ihnen verlor Wien nicht nur immens bedeutende Wissenschaftler (sie werden als ,Erfinder' der Radiochemie, Diskursanalyse und Friedens-

\footnotetext{
${ }^{36}$ Vgl. NeMEC, TASCHWER, Terror 154. Die Studierendenzahl verringerte sich von 1918 bis 1923 generell um ca. 2000 bis 3000 Hörerinnen und Hörer.

${ }^{37} \mathrm{Vgl}$. Diener, Bericht 6, 14.

${ }^{38} \mathrm{Vgl}$. PrOCHASKA, Leidensweg $1 \mathrm{f}$.
}

und Solidaritätsforschung bezeichnet), sondern auch zwei Nobelpreisträger: Bárány (1914), Landsteiner (1930). Sie waren wegen ihres jüdischen Hintergrunds schlecht behandelt worden und kehrten der Universität den Rücken: Bárány wechselte an die Universität Uppsala, Landsteiner an das Rockefeller-Institut in New York und Pauly an die ETH Zürich, später nach Princeton. 1938 waren drei weitere Nobelpreisträger zur Auswanderung gezwungen: Franz Viktor Hess (1936), Otto Löwi (1936) und Erwin Schrödinger (1933). 1930 hatte „unzweifelhaft der bedeutendste Rechtslehrer der Wiener Universität" Hans Kelsen, der Schöpfer der Verfassungsgerichtsbarkeit, Wien verlassen. In einem scharfzüngigen Bericht bezeichnete es die "Arbeiter-Zeitung“ als einen "Skandal“, "daß der Unterrichtsminister [Heinrich Srbik] ihn ziehen läßt." „Die Kelsen gehen, die Gleispach bleiben ...", hieß es in der "AZ“ in Anspielung an den Proto-Nazi und Rektor Wenzel Gleispach, der 1930 eine rassistische Studentenordnung einführte. ${ }^{39}$

Indes lesen sich die von den Wiener Rektoren Jahr für Jahr aufgelisteten Namen der durch Berufungsabwehr Gehaltenen und neu Berufenen wie ein Who is Who später pronocierter nationalsozialistischer bzw. NS-naher Wissenschaft in Österreich. Abgewehrt wurden die Wegberufungen u.a. von Rudolf Egger, Wenzel Gleispach, Alexander Hold-Ferneck, Hermann Junker, Oswald Menghin, Richard Meister, Carl Ludwig Patsch, Othmar Spann, Heinrich Srbik und Friedrich Wild. Zu Professoren wurden u.a. Alexander Hold-Ferneck, Karl Gottfried Hugelmann, Dietrich Kralik, Robert Lach, Oswald Menghin, Rudolf Pöch, Otto Reche, Othmar Spann, Heinrich Srbik und Jan Versluys berufen. Wie Klaus Taschwer zeigt, versammelten sich Geisteswissenschaftler in einer konspirativen Professorenvereinigung namens „Bärenhöhle“,

${ }^{39}$ ANONYM, Die Kelsen gehen 2. 
die zwischen 1925 und 1933 die Habilitierung zahlreicher hochqualifizierter Jungwissenschaftlerinnen und Jungwissenschaftler aus politischen und "rassischen" Motiven verhinderte und damit für den Ausschluss der Talente hauptverantwortlich war. ${ }^{40}$ Taschwer nennt auch eine Reihe von gescheiterten Habilitationswerbern, u.a. die Physiker Karl Horovitz und Otto Halpern, den Albanologen Maximilian Lambertz, den Philosophen Edgar Zilsel sowie Leonore Brecher und Paul Weiss, Schüler Hans Leo Przibrams, des Zoologen und Gründers der Biologischen Versuchsanstalt der Akademie der Wissenschaften im Prater. ${ }^{41}$ Bekannt sind weiters die verhinderten, gescheiterten oder nicht zustande gekommenen Habilitationen u.a. der Ökonomen Fritz Machlup, Paul RosensteinRodan und Martha Stefanie Braun, ${ }^{42}$ der Wirtschaftsforscher Franz Nemschak und Josef Steindl, des Philosophen Karl Popper, der Orientalisten Gustav Gruenebaum und Karl Mlaker, der Kunsthistoriker Otto Benesch und Otto Pächt, des Historikers Peter Kuranda, des Germanisten Franz H. Mautner sowie des Chemikers Hans Rudolf Frisch und des Physikers Franz Urbach. ${ }^{43}$

Davon legte letzterer zu Silvester 1934 Zeugnis ab: „Als ich meine Studien (1921) begann, war in Österreich die akademische Laufbahn für einen Juden zwar etwas erschwert, aber durchaus zugänglich; als ich sie beendete (1926, also lange vor Ausbruch des deutschen Nationalsozialismus) war sie praktisch bereits fast unmöglich, und zwei Jahre nach dem Doktorat, als ich habilitationsreif war, war [daran] nicht mehr zu denken. Es war damals eine staatliche Anstellung für einen Juden so gut wie unerreichbar geworden, und besonders die Universität und technische Hochschule waren Zentren des

\footnotetext{
${ }^{40}$ TASCHWER, Geheimsache Bärenhöhle [im Druck].

41 Vgl. ebd.

${ }^{42}$ Vgl. KLAusinger, Academic Anti-Semitism 191-204.

${ }^{43}$ Vgl. u.a. Korrespondenzen, Personal Files, SPSL.
}

schärfsten Antisemitismus. Ich war so dem lautlosen österreichischen Hochschulantisemitismus ausgeliefert." 44

Auch Erich Voegelin erinnerte sich, dass schon ,mit der Ausrufung der Republik der Antisemitismus zu einem allgegenwärtigen Phänomen an der Wiener Universität" geworden war: Voegelin sprach dezidiert von einer „Blockade“ der "ausgezeichneten“ ",jüngeren jüdischen Wissenschaftler", die sie in oft wissenschaftsferne Brotberufe verdrängte. ${ }^{45}$ Nur wenige konnten sich durch außeruniversitäre Forschung ihren Lebensunterhalt verdienen, u.a. in der Biologischen Versuchsanstalt der Akademie der Wissenschaften im Prater, am Psychologischen Institut der Gemeinde Wien und seiner Wirtschaftspsychologischen Forschungsstelle sowie am Österreichischen Institut für Konjunkturforschung. Manche fanden Stellen an Museen, Bibliotheken und Archiven oder waren selbständig tätig. Andere wie z.B. Karl Popper wählten die Schullaufbahn: "I understood that circumstances in Austria could not permit me to obtain an official position at the university. I therefore decided to prepare myself for a post at public school." 46

Viele diese Talente verließen Österreich. Physiker (wie Karl Horovitz und Otto Halpern), Chemiker (wie Max Perutz), Biologen (wie Paul Weiss), empirische Sozialforscher (wie Paul Lazarsfeld und Marie Jahoda), Konjunkturforscher (wie Friedrich August Hayek und Oskar Morgenstern), Kunsthistoriker (wie Ernst Gombrich, Otto Kurz und Otto Pächt) und Vertreter neuer Strömungen in der Philosophie (wie Karl Popper, Herbert Feigl und Friedrich Waismann) fanden Stellen in England und Amerika. ${ }^{47}$ Hans Kelsen ebnete manchen seiner Schüler, deren Laufbahn an der Wiener Rechts- und

\footnotetext{
${ }^{44}$ Franz Urbach an Esther Simpson, 31. 12. 1934, SPSL $342 / 5$.

45 VOEgELIN, Autobiographische Reflexionen 24.

${ }^{46}$ POPPER, Details of Educational Career.

${ }^{47} \mathrm{Vgl}$. Feichtinger, Wissenschaft.
} 
Staatswissenschaftlichen Fakultät blockiert war, ${ }^{48}$ den Weg ins Ausland, und das längst vor dem "Anschluss" 1938.49 Der Privatdozent Josef Laurenz Kunz, in Wien Mitglied im Kreis um Kelsen, ${ }^{50}$ weilte 1932/33 als Rockefeller Fellow in den USA, bevor er 1934 eine dauerhafte Stelle an die University of Toledo in Ohio fand. Auch die beiden Assistenten Kelsens in Köln - Erich Hula und Leo Gross - verdankten ihre wissenschaftlichen Laufbahnen wesentlich der Rockefeller grant policy. Die Wiener Kelsen-Schüler verließen - so wie Kelsen - Köln nach der Machtübernahme Hitlers 1933 in verschiedene Richtung: der langjährige Rockefeller Fellow Leo Gross wurde auf Vorschlag der Stiftung 1940 zunächst auf eine Professor an die New School for Social Research in New York berufen. Der ebenfalls langjährige Rockefeller Fellow Erich Hula hatte 1933 das Angebot der New School for Social Research in New York zurückgewiesen und war als Arbeiterkammersekretär nach Graz zurückgekehrt. Nach dem "Anschluss“ nahm er die von der Stiftung geförderte Professur für Politikwissenschaft und Völkerrecht an der New School schließlich an. Die Rockefeller Stiftung ebnete auch anderen Mitgliedern des außeruniversitären Kreises um Hans Kelsen den Weg zu einer akademischen Berufslaufbahn in den USA: Hans Ernst Fried, Josef Herbert Fürth, Felix Kaufmann, Alfred Schütz und Erich Voegelin. Mit Ausnahme von Voegelin hatte keiner in Österreich eine Universitätsstelle inne gehabt. ${ }^{51}$

Die Universität Wien hatte Abweichler - die so genannten "Asiaten“ - vor die Tür gesetzt. Zugleich hatte sie aber den Verlust ihrer systemisierten Lehrkräfte auf die Notlage der Universität zurückgeführt. Die "wirkliche Not unserer Hochschulen" hatte aber andere Ursachen als

\footnotetext{
${ }^{48}$ Vgl. ReIter-ZatLouKal, Antisemitismus 191-198.

${ }^{49}$ Vgl. EHS, Vertreibung 147-174.

50 WALTER, JABLONER, ZELENY, Kreis.

${ }^{51}$ Vgl. Feichtinger, Vernetzungen 321-338. FLECK, Bereicherungen 167-180, $208 f$.
}

gekürzte Universitätsbudgets. In einem spitzen Zeitungskommentar anlässlich eines Memorandums der Universitäten über ihre Notlage von 1925 heißt es: „Dieselben Herren, die heute ihre Unterschrift unter die Memoranden setzen, dieselben Herren sind verantwortlich für jenen Zelotengeist, der seit einem halben Dezennium sich gerade an den Stätten der freien Forschung festgesetzt hat. [...] Unter ihrer Patronanz habe sich eine Minorität unter den Studierenden als Terrorgruppe etabliert, mit der weithin sichtbaren Tendenz, die Rasseneinheit unserer Hochschulen zu schützen. Dieselben Herren, die bei der Berufung von Lehrkräften sich nicht etwa von der wissenschaftlichen Qualität, sondern den Stammbaum kontrollieren und mit dem Eifer, der einer besseren Sache wert wäre, nach jüdischen Vorfahren fahnden, dürften nicht davon sprechen, daß die Hochschulen in Not seien und daß ihnen geholfen werden soll. Bevor diesen Funktionären nicht beigebracht würde, daß Forschung, Wahrheit und Wissenschaft nichts mit Antisemitismus zu tun habe, so lange hätten sie keinen Anspruch darauf, ernst genommen zu werden, wenn sie sich über die Gefährdung ihrer Arbeit beklagten. ${ }^{52}$

Der Antisemitismus ließ nicht nach:53 Vor 1933 wurden pronociert deutsch-völkische Professoren, die Wien vor und nach 1918 verlassen hatten, wieder auf Lehrstühle der Universität Wien zurückberufen. Die Geografen Fritz Machatschek (1928 von der ETH Zürich) und Hugo Hassinger (1931 von der Universität Freiburg im Breisgau) sollten in Wien federführend am Aufbau der "Südostdeutschen Forschungsgemeinschaft" mitwirken, einer Organisation, deren Aufgabe in wissenschaftlichen Vorarbeiten zur nationalsozialistischen „Umvolkung“ bestand.

Mit Anbruch der Dollfuß-Schuschnigg-Diktatur (1933/34) sind im Hinblick auf den Österreich

\footnotetext{
52 O. B., Not 7.

${ }^{53}$ STADLER, Antisemitismus 207-237.
} 
bezogenen Braindrain drei Bewegungen von Wiener Forschern zu vermerken:

- Erstens, von Deutschland nach Österreich: Vor der Machtübernahme Hitlers wurde z.B. der bedeutende Kunststoffchemiker Hermann Mark, der von den Nationalsozialisten als Mischling verfolgt wurde, auf den Chemielehrstuhl Wegscheiders berufen. Mark, ein Dollfuß-Freund, beschäftigte in Wien drittmittelfinanziert zahlreiche Talente, unter ihnen den späteren Nobelpreisträger Max Perutz (1962), der 1936 Wien verließ. Mark selbst emigrierte 1938 in die USA, wo er das Institute of Polymer Research in New York aufbaute;

- Zweitens, von Österreich nach HitlerDeutschland: Im autoritären Ständestaat wurden nationalsozialistisch orientierte Professoren suspendiert bzw. in den Ruhestand versetzt; an der Universität Wien u.a. der Rechts- und Staatswissenschaftler Karl Gottfried Hugelmann, der Jurist Wenzel Gleispach, der Paläontologe Othenio Abel, der Geograph Friedrich Machatschek, der Orientalist Viktor Christian, der Germanist Rudolf Much sowie die Historiker Carl Ludwig Patsch, Viktor Bibl und Hans Übersberger. Manche von ihnen wurden sogleich auf Lehrstühle in Hitler-Deutschland berufen: Hugelmann an die Universität Münster, Gleispach an die Universität Berlin, Abel an die Universität Göttingen, Machatschek an die Universität München und Übersberger an die Universität Breslau;

- Drittens, von Österreich in die weite Welt: 1934 und danach verloren nicht nur nationalsozialistisch, sondern auch demokratisch gesinnten Professoren ihre Stellung. Bemerkenswert ist, dass - wie Klaus Taschwer zeigt - von den 19 Wiener Universitätsprofessoren, die vorzeitig pensioniert wurden, 11 jüdischer Herkunft waren. ${ }^{54}$ Manche von ihnen verließen bald das Land. Prominente Beispiele dafür sind u.a. Juli-

${ }^{54} \mathrm{Vgl}$. TASCHWER, Niedergang. us Tandler und Heinrich Gomperz. Der Anatomieprofessor Tandler hatte seit 1918 als Jude und als sozialdemokratischer Wiener Stadtrat den Hass der christlichsozialen und deutschnationalen Machthaber an der Universität auf sich gezogen. 1934 wurde er verhaftet, an der Universität zwangspensioniert, bevor er im gleichen Jahr in Moskau Zuflucht fand. Auch der Philosoph Gomperz, der seit 1924 einen der vier Philosophielehrstühle innehatte, verließ Österreich und übersiedelte 1935 an die University of Southern California in Los Angeles.

Viele als Juden eingestufte Forscherinnen und Forscher verblieben aber in Österreich, solange es ging. Der „Rückzug im faulen Kompromiß", 55 so Hilde Spiel, sei ein bequemer Weg gewesen, sich mit der Schuschnigg-Diktatur zu arrangieren. Mit dem „Anschluss“ 1938 war dieser Weg jedoch verstellt und die Vernunft der systematischen Vertreibung preisgegeben.

\section{Ergebnis}

In dieser Abhandlung wurde $\mathrm{zu}$ zeigen versucht, dass der Braindrain aus Österreich nicht erst mit der NS-Machübernahme 1938, sondern schon bald nach 1918 einsetzte. Viele junge Talente verließen dauerhaft das Land. Der rassische Antisemitismus, der Abbau akademischer Stellen und ein wissenschaftsfeindliches Klima hatte ihnen den Eintritt in die akademische Laufbahn versperrt. Manche blieben, sie wirkten häufig außerhalb der Universität, die zusehends an internationalem Ansehen verlor. Die in der Zwischenkriegszeit berufenen Wiener Universitätsprofessoren, auf deren Werk heute noch zurückgegriffen wird, lassen sich fast an einerHand abzählen. $\mathrm{Zu}$ nennen wären u.a. Hans Kelsen, Karl Bühler, Moritz Schlick, Hermann Mark, und Nikolai Turbetzkoy. Waren sie nicht verstorben oder ausgewandert, so wurden auch

${ }^{55}$ Vgl. SPIEL, Die hellen und die finsteren Zeiten 115. 
sie nach dem „Anschluss“ 1938 Opfer politischer und "rassischer" Verfolgung. Die meisten konnten flüchten. Während die Aufnahmeländer deren wissenschaftliche Kompetenz als großen Gewinn erachteten, wurde deren Ausschluss und Vertreibung auch in der jungen Zweiten Republik nicht als Verlust verbucht. Allein in dieser Haltung zeigt sich, dass der schon 1918 eingeschlagene Weg der Universitäten in die Provinzialität nach 1945 eine Zeitlang konsequent weiter beschritten wurde.

\section{Korrespondenz:}

Doz. Dr. Johannes Feichtinger

Österreichische Akademie der Wissenschaften

Institut f. Kulturwissenschaften $u$. Theatergeschichte

Postgasse 7/4

1010 Wien

johannes.feichtinger@oeaw.ac.at

\section{Abkürzungen:}

SPSL Archiv der Society for the Protection of Science and Learning, Bodleian Library Oxford

\section{Literatur:}

ANONYM, Die Koalition gegen die Universität, in: Arbeiter-Zeitung, Nr. 76 v. 18.3.1921 (Morgenblatt) 2 .

ANONYM, Die Erhaltung der Wiener Universität, in: Wiener Abendpost, Nr. 263 v. 19. 11. 1921 (Beilage zur Wiener Zeitung) 3.

ANONYM, Drohungen gegen Rektor Dr. Diener. Der beleidigte Verein zur Erhaltung der Universität, in: Reichspost, Nr. 328 v. 8. 12. 1922 (Morgenblatt) 6.

ANONYM, Rückgang der Frequenzziffer an unseren Universitäten, in: Reichspost, Nr. 294 v. 1. 8. 1919 (Morgenblatt) 6-7.

ANONYM, Offener Brief der deutschen Studentenschaft der Hochschulen Wiens. Wien, 18. Dezember 1922, in: Deutschösterreichische Tageszeitung. Unabhängiges Blatt für völkische Politik, Nr. 340 v. 20. 12. 1922, 5.

ANONYM, Antwort des akademischen Senats auf das deutsch-nationale Memorandum. Keine Grundlage für Beratungen im Senat, in: Neue Freie Presse, Nr. 20937 v. 21. 12. 1922 (Morgenblatt) 1.

ANONYM, Der Bundeskanzler über die Forderungen der antisemitischen Studentenschaft. Ein Protest der Wiener Kultusgemeinde, in: Neue Freie Presse, Nr. 20919 v. 3. 12. 1922 (Morgenblatt) 6.

ANONYM, Die Kelsen gehen, die Gleispach bleiben, in: in: Arbeiter-Zeitung, Nr. 189 v. 11. 7. 1930, 2-3.

Mitchell G. AsH, Jüdische Wissenschaftlerinnen und Wissenschaftler an der Universität Wien von der Monarchie bis nach 1945. Stand der Forschung und offene Fragen, in: Oliver RATHKOLB (Hg.), Der lange Schatten des Antisemitismus. Kritische Auseinandersetzung mit der Geschichte der Universität Wien im 19. und 20. Jahrhundert (= Zeitgeschichte im Kontext 8, Wien 2013) 93-122.

O. B., Die wirkliche Not unserer Hochschulen, in: Der Morgen. Wiener Montagblatt, Nr. 45 v. 9. 11. 1925, 7.

Friedrich Johann BECKE, Bericht über das Studienjahr 1918/19, in: Die feierliche Inauguration des Rektors der Wiener Universität für das Studienjahr 1919/20 (Wien 1919) 3-78. 
Karl DiEnER, Das Memorandum der deutschen Studentenschaft, in: Reichspost, Nr. 303 v. 10. 12. 1922 (Morgenblatt) 1.

Karl DiENER, Die Vorgänge an den Wiener Hochschulen, in: Deutschösterreichische Tageszeitung. Unabhängiges Blatt für völkische Politik, Nr. 332 v. 12. 12. $1922,1-2$.

Karl DiENER, Bericht über das Studienjahr 1922/23, in: Die feierliche Inauguration des Rektors der Wiener Universität für das Studienjahr 1923/24 (Wien 1923) 3-43.

Alphons DOPSCH, Bericht über das Studienjahr 1920/21, in: Die feierliche Inauguration des Rektors der Wiener Universität für das Studienjahr 1921/22 (Wien 1921) 3-52.

Tamara EHS, Vertreibung in drei Schritten. Hans Kelsens Netzwerk und die Anfänge österreichischer Politikwissenschaft, in: Österreichische Zeitschrift für Geschichtswissenschaften 21, 2 (2010) 147-174.

Johannes FEICHTINGER, Verletzte Autonomie. Wissenschaft und ihre Struktur in Wien 1848 bis 1938, in: Friedrich STADLER, Elisabeth NEMETH, Herbert POSCH, Katharina KNIEFACZ (Hgg.), Universität Forschung - Lehre (= 650 Jahre Universität Wien Aufbruch ins neue Jahrhundert 1, Wien 2015) [im Druck].

Johannes FEICHTINGER, Transatlantische Vernetzungen. Der Weg Hans Kelsens und seines Kreises in die Emigration, in: Robert WALTER, Werner OGRIS, Thomas OlechOWSKI (Hgg.), Hans Kelsen. Leben - Werk - Wirkung (= Schriftenreihe des Hans Kelsen-Instituts 32, Wien 2009) 321-338.

Johannes FEICHTINGER, Wissenschaft zwischen den Kulturen. Österreichische Hochschullehrer in der Emigration 1933-1945 (= Campus Forschung 816, Frankfurt am Main-New York 2001).

Silke FENGLER, Günther LuXBACHER, „Aufrechterhaltung der gemeinsamen Kultur“. Die Deutsche Forschungsgemeinschaft und die ÖsterreichischDeutsche Wissenschaftshilfe in der Zwischenkriegszeit, in: Berichte zur Wissenschaftsgeschichte 4 (2011) 303-328.

Christian FLECK, Autochthone Provinzialisierung. Universität und Wissenschaftspolitik nach dem Ende der nationalsozialistischen Herrschaft in Österreich, in: Österreichische Zeitschrift für Geschichtswissenschaft 7 (1996) 67-92.

Christian FLECK, Transatlantische Bereicherungen. Zur Erfindung der empirischen Sozialforschung (Frankfurt am Main 2007) 167-180.

Walter HÖFLECHNER, Wissenschaft, Hochschule, Staat in Österreich bis 1938, in: Christian BRÜNNER, Helmut KONRAD (Hgg.), Die Universität und 1938
(= Böhlaus zeitgeschichtliche Bibliothek 11, WienKöln 1989) 57-74.

Matthäus KATTINGER, Österreichs verheerender Migrationssaldo, in: Neue Zürcher Zeitung, Nr. 76 v. 1. 4. 2014, 25.

Hans Kelsen, Die Not der Universität, in: Neue Freie Presse, Nr. 20409 v. 24. 6. 1921 (Morgenblatt) 1.

Hansjörg KlaUsinger, Academic Anti-Semitism and the Austrian School. Vienna, 1918-1945, in: Atlantic Economic Journal 42, 2 (2014) 191-204.

Ota KONRÁD, Die Geisteswissenschaften nach den Umbruchsjahren 1938 und 1938. Die Deutsche Universität in Prag und die Universität in Wien im Vergleich, in: Sabine SCHLEIERMACHER, Udo SCHAGEN (Hgg.), Wissenschaft macht Politik. Hochschule in den politischen Systembrüchen 1933 und 1945 (= Wissenschaft, Politik und Gesellschaft 3, Stuttgart 2009) 193-218.

Brigitte LichtENBERGER-FENZ, „....Deutscher Abstammung und Muttersprache". Österreichische Hochschulpolitik in der Ersten Republik (=Veröffentlichungen des Ludwig-Boltzmann-Institutes für Geschichte der Gesellschaftswissenschaften 19, Wien-Salzburg 1990).

M. ST., Die obenan sind. Die Besuchsziffern der Wiener Universität, in: Reichspost, Nr. 306 v. 13.8. 1919 (Morgenblatt) 7.

Birgit NemEC, Klaus TAschwer, Terror gegen Tandler. Kontext und Chronik der antisemitischen Attacken am I. Anatomischen Institut der Universität Wien, 1910 bis 1933, in: Oliver RATHKOLB (Hg.), Der lange Schatten des Antisemitismus. Kritische Auseinandersetzung mit der Geschichte der Universität Wien im 19. und 20. Jahrhundert (= Zeitgeschichte im Kontext 8, Wien 2013) 147181.

Karl POPPER, Details of Educational Career and University Training and Decrees and Teaching Experience (ungedrucktes Manuskript, SPSL 319/4).

Herbert PosCH, Doris INGRISCH, Gerd DRESSEL (Hgg.), "Anschluß" und Ausschluss 1938. Vertriebene und verbliebene Studierende der Universität Wien (= Emigration - Exil - Kontinuität. Schriften zur zeitgeschichtlichen Kultur- und Wissenschaftsforschung 8, Wien-Berlin 2008).

Herbert PosCH, Studierende und die Universität Wien in der Dauerkrise 1918 bis 1938, in: DERS., Doris INGRISCH, Gerd DRESSEL (Hgg.), "Anschluß“ und Ausschluss 1938. Vertriebene und verbliebene Studierende der Universität Wien (= Emigration Exil - Kontinuität. Schriften zur zeitgeschichtlichen Kultur- und Wissenschaftsforschung 8, Wien-Berlin 2008) 61-97. 
Bruno PROCHASKA, Der Leidensweg zum Doktorhut. Aus dem Daseinskampf der Wiener Studenten, in: Neues Wiener Tagblatt, Nr. 26 v. 27. 6. 1925, 1-2.

Oliver RATHKOLB, Gewalt und Antisemitismus an der Universität Wien, in: DERS. (Hg.), Der lange Schatten des Antisemitismus. Kritische Auseinandersetzung mit der Geschichte der Universität Wien im 19. und 20. Jahrhundert (= Zeitgeschichte im Kontext 8, Wien 2013) 69-92.

JOSEF REDLICH, Über die Situation für jüdische Gelehrte an den österreichischen Universitäten, in: Oliver Rathkolb, Gewalt und Antisemitismus an der Universität Wien und die Badeni-Krise 1897. Davor und danach, in: Oliver RATHKOLB (Hg.), Der lange Schatten des Antisemitismus. Kritische Auseinandersetzung mit der Geschichte der Universität Wien im 19. und 20. Jahrhundert (= Zeitgeschichte im Kontext 8, Wien 2013) 275-315 (= Vorlage zur Rede).

Ilse ReITER-ZATLOUKAL, Antisemitismus und Juristenstand. Wiener Rechts- und Staatswissenschaftliche Fakultät und Rechtspraxis vom ausgehenden 19. Jahrhundert bis zum "Anschluss“ 1938, in: Oliver RATHKOLB (Hg.), Der lange Schatten des Antisemitismus. Kritische Auseinandersetzung mit der Geschichte der Universität Wien im 19. und 20. Jahrhundert (=Zeitgeschichte im Kontext 8, Wien 2013) 183-205.

Emil REISCH, Aufgaben unserer Universitäten nach dem Kriege. Inaugurationsrede gehalten von Dr. Emil Reisch, Rektor der K.K. Universität, in: Die feierliche Inauguration des Rektors der Wiener Universität (Wien 1917) 57-96.

Ernst SCHWIND, Bericht über das Studienjahr 1919/20, in: Die feierliche Inauguration des Rektors der Wiener Universität für das Studienjahr 1920/21 (Wien 1920) 3-60.

E[rnst] SCHWIND, Die Berufungen österreichischer Gelehrter an deutsche Universitäten, in: Neue Freie Presse, Nr. 2040 v. 19.6.1921 (Morgenblatt) 1

Ignaz SEIPEL, Die Kulturpolitik der ChristlichSozialen, in: Reichspost, Nr. 263 v. 23.9.1920 (Morgenblatt) 1-2.

Hans SPERL, Die Wiedereinführung des Kollegiengeldes, in: Neue Freie Presse, Nr. 19866 v. 17. 12. 1919 (Morgenblatt) 3.
Hans SPERL, Bericht über das Studienjahr 1924/25 [Kundgebung], in: Die feierliche Inauguration des Rektors der Wiener Universität für das Studienjahr 1925/26 (Wien 1925) 3-45.

Hilde SPIEL, Die hellen und die finsteren Zeiten. Erinnerungen 1911-1946 (Reinbek 1991).

Friedrich STADLER (Hg.), Vertriebene Vernunft. Emigration und Exil österreichischer Wissenschaft 1930-1940. Band 1 (= Emigration - Exil - Kontinuität. Schriften zur zeitgeschichtlichen Kultur- und Wissenschaftsforschung 1, Wien-Berlin 2004, Original 1987-1988) 9-41.

Friedrich STADLER, Antisemitismus an der Philosophischen Fakultät der Universität Wien. Am Beispiel von Moritz Schlick und seines Wiener Kreises, in: Oliver RAthKOlb (Hg.), Der lange Schatten des Antisemitismus. Kritische Auseinandersetzung mit der Geschichte der Universität Wien im 19. und 20. Jahrhundert (= Zeitgeschichte im Kontext 8, Wien 2013) 207-237.

Klaus TASCHWER, Geheimsache Bärenhöhle. Wie ein antisemitisches Professorenkartell der Universität Wien nach 1918 jüdische und linke Forscherinnen und Forscher vertrieb, in: Regina FRITZ, Grzegorz RossolinSKI-LieBE, Béla RÁSKY (Hg.), Alma mater antisemitica. Akademisches Milieu, Juden und Antisemitismus an den Universitäten Europas zwischen 1918 und 1939 (Wien 2015) [im Druck].

Klaus TASCHWER, Niedergang der Universität Wien in der ersten Hälfte des 20. Jahrhunderts (ungedrucktes Buchmanuskript, in das der Autor Einblick nehmen durfte).

Eric VOEGELIN, Autobiographische Reflexionen, hg. v. Peter J. OpITZ in Verbindung mit dem EricVoegelin-Archiv (München 1994).

Robert WaLter, Clemens Jabloner, Klaus Zeleny (Hgg.), Der Kreis um Hans Kelsen. Die Anfangsjahre der Reinen Rechtslehre (= Schriftenreihe des Hans Kelsen-Instituts 30, Wien 2008).

Erika WEINZIERL, Hochschulleben und Hochschulpolitik zwischen den Kriegen, in: Norbert LeSER (Hg.), Das geistige Leben Wiens in der Zwischenkriegszeit (= Quellen und Studien zur österreichischen Geistesgeschichte im 19. und 20. Jahrhundert 1, Wien 1981) 72-85.

R[ichard] WeTtSTEIN, Die Hilfsaktionen für die Wissenschaft, in: Neue Freie Presse, Nr. 20404 v. 19. 6. 1921 (Morgenblatt) 2. 